\title{
ESTUDO FOURIER: EVOLOCUMABE E DESFECHOS CLÍNICOS EM PACIENTES COM DOENÇA CARDIOVASCULAR
}

\section{EVOLOCUMAB AND CLINICAL OUTCOMES IN PATIENTS WITH CARDIOVASCULAR DISEASE}

Pedro Henrique Pedruzzi Segato1; Lucas Wagner Görtz"; Mauricio Carvalho1

\section{Dr. Eugene Braunwald is quoted saying "This is like the era of the statins coming in. It's a new ballgame."}

O uso de estatinas e a consequente diminuição do colesterol LDL comprovadamente reduz eventos cardiovasculares. Esse efeito é ainda maior quando tais medicamentos são associados, por exemplo, a um inibidor de absorção do colesterol. O estudo IMPROVE-IT, que utilizou uma associação de estatinas com ezetimibe, diminuiu os níveis médios de colesterol LDL na população estudada para $54 \mathrm{mg} /$ dl (os menores até então) e comprovou seu benefício. Nesse cenário, a busca por novas terapêuticas a fim de reduzir ainda mais os níveis de colesterol LDL propiciou o estudo FOURIER, que teve como objetivo avaliar o evolocumabe.

O evolocumabe é um anticorpo monoclonal que inibe a pró-proteína convertase subtilisina/kexina tipo 9 (PCSK9), responsável pela degradação de receptores de LDL (LDL-r) na superfície do hepatócito. 0 efeito final é um aumento da expressão de LDL-r com consequente redução dos níveis séricos de LDL.

Os inibidores da PCSK9 têm emergido como uma classe de medicamentos que efetivamente reduzem os níveis de LDL colesterol em aproximadamente 60\%. Estudos genéticos mostraram que a perda de alelos que inibem a função da PCSK9 reduziu as taxas de infarto agudo do miocárdio (IAM). Além disso, outros estudos de fase 2 e 3 também com inibidores da PCSK9 demonstraram redução do risco cardiovascular, porém com um número baixo de eventos registrados (apenas 100).
Para comprovar o efeito positivo dos inibidores da PCSK9, o FOURIER testou a eficácia e segurança do evolocumabe associado à terapia com estatinas de moderada/alta-intensidade em pacientes com doença cardiovascular aterosclerótica clinicamente evidente.

Foi um estudo duplo-cego, randomizado, placebocontrolado e multicêntrico. Foram comparados, de forma prospectiva, dois grupos de pacientes, os quais foram divididos conforme demonstrado na figura 1.0 tempo médio de seguimento foi de 2,2 anos em um total de 27.564 pacientes de 49 países diferentes.

Figura 1: Desenho do Estudo

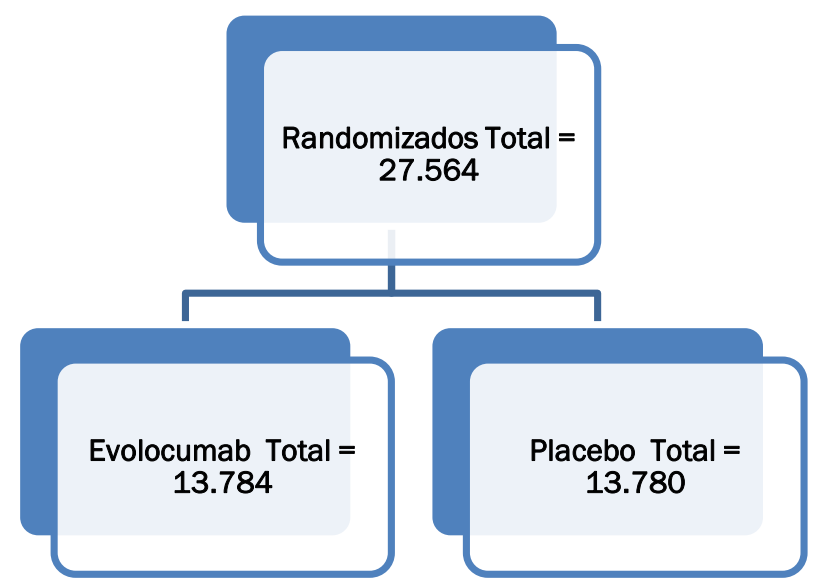

1- Complexo Hospital de Clínicas / Universidade Federal do Paraná, Curitiba, Paraná, Brasil.

\author{
Contato do Autor / Mail to: \\ Pedro Henrique Pedruzzi Segato- segatopedro@yahoo.com.br \\ Rua General Carneiro, 181, Alto da Glória, Curitiba, Paraná. CEP 80060-900
}




\section{REVISTA MÉDICA DA UFPR}

Foram incluídos no estudo homens e mulheres entre 40-85 anos com evidência clínica de doença cardiovascular aterosclerótica (IAM, acidente vascular cerebral não hemorrágico [AVCi] e doença arterial obstrutiva periférica [DAOP] sintomática) e alto risco cardiovascular.

Todos os pacientes deveriam ter um colesterol LDL em jejum $\geq 70 \mathrm{mg} / \mathrm{dl}$ ou não-HDL $\geq 100 \mathrm{mg} / \mathrm{dl}$, triglicerídeos $\leq 400 \mathrm{mg} / \mathrm{dl}$ e estar sob tratamento otimizado com estatinas de moderada/alta intensidade, definido como no mínimo atorvastatina $20 \mathrm{mg}+/$ - ezetimibe, ou terapêutica equivalente. A dose do evolocumabe foi de $140 \mathrm{mg}$ a cada 2 semanas ou 420 mg em dose única mensal, subcutânea.

Os dados foram coletados pelo patrocinador do estudo (Amgen $\AA$ ) e analisado pelo mesmo grupo do estudo Trombólise no Infarto Agudo do Miocárdio (TIMI).

Dentre todos os pacientes eleitos para o estudo, 81,1\% apresentavam história prévia de IAM, 19,4\% de AVCi e $13,2 \%$ de DAOP. Além disso, $69,3 \%$ deles estavam sob uso de terapia de alta intensidade com estatinas e $29,7 \%$ com terapia de moderada intensidade. As terapias secundárias mais utilizadas pelos participantes do estudo foram os antiagregantes plaquetários (92,3\%), iECA/BRA/espironolactona $(78,2 \%)$ e os $\beta$-bloqueadores (75,6\%). A média de idade em ambos os grupos foi de 62,5 anos e 3/4 dos participantes eram homens, a maioria de raça branca $(85 \%)$

O objetivo primário do estudo foi um composto de morte por causas cardiovasculares, AVC, IAM, hospitalização por angina instável e revascularização coronariana. Os três objetivos secundários foram: morte por evento cardiovascular, AVC e IAM.

De início, a população estudada apresentava níveis médios de $L D L$ de $92 \mathrm{mg} / \mathrm{dl}$, os quais foram reduzidos em $56 \mathrm{mg} / \mathrm{dl}$ - valor médio absoluto. Cabe destacar que esse efeito foi mantido ao longo do tempo no grupo evolocumabe, atingindo o alvo terapêutico já dentro das primeiras 4 semanas de tratamento, conforme demonstrado na figura 2.

O evolocumabe manteve níveis absolutos de LDL $<70 \mathrm{mg} / \mathrm{dl} \mathrm{em} 87 \%$ dos pacientes, $<40 \mathrm{mg} / \mathrm{dl} \mathrm{em} 67 \%$ deles, e $<25 \mathrm{mg} / \mathrm{dl}$ em $42 \%$ dos pacientes do grupo, com significância estatística quando comparado ao grupo placebo $(p<0,001)$. Além disso, apresentou efeito adicional de redução dos níveis séricos de colesterol não-HDL em $52 \%$ e da apoliproproteína B (proteína transportadora de LDL para os tecidos) em $49 \%$.

Em relação aos desfechos cardiovasculares primários agrupados, 1388 pacientes apresentaram tais eventos no grupo evolocumabe (9,8\%) versus 1563 pacientes no grupo placebo (11,3\%), redução de $15 \%$ no risco de incidência (hazard ratio $=0,85 ; 95 \% \mathrm{Cl}$ - 0,79 a 0,92; $\mathrm{p}<0,001$ ). Estima-se que esse efeito seja cumulativo ao longo do tempo, uma vez que no $1^{\circ}$ e no $2^{\circ}$ ano de acompanhamento esses desfechos foram 12\% e 19\% menos frequentes, respectivamente.
Da mesma forma, o evolocumabe reduziu os desfechos cardiovasculares secundários em $20 \%$, quando comparado ao grupo placebo (816 x 1013 pacientes), também com efeito benéfico cumulativo ao longo do tempo: $16 \%$ de redução no $1^{\circ}$ ano e $25 \%$ redução no $2^{\circ}$ ano de seguimento (hazard ratio=0,80; 95\% Cl- 0,73 a 0,88; $p<0,001)$.

A respeito dos desfechos analisados separadamente, o evolocumabe reduziu taxas de IAM em $27 \%$, AVCi em $25 \%$ e revascularização coronariana em $22 \%$, valores estatisticamente significativos quando comparados ao grupo placebo $(p<0,001)$.

No entanto, cabe ressaltar que a droga não reduziu mortes por causas cardiovasculares (hazard ratio=1,05 [0,88-1,25]), revascularização por angina instável (hazard ratio=0,99 [0,82-1,18]), internamento por IC descompensada (hazard ratio $=0,98[0,8-1,13]$ ) ou morte por qualquer causa (hazard ratio=1,04 [0,91-1,19])

Todos os benefícios do evolocumabe foram mantidos independentemente do quartil em que os níveis de LDL dos pacientes se encontravam no começo do estudo (Q1<80mg/dl; Q2 $80-<92 \mathrm{mg} / \mathrm{dl} ; \quad$ Q3 $92-109 \mathrm{mg} / \mathrm{dl}$; Q4>109mg/dl), com benefício também cumulativo ao longo do tempo observado e a despeito do uso prévio de estatinas e/ou ezetimibe.

Não foi verificada diferença significativa nos efeitos adversos entre os dois grupos ao longo do tempo. Além disso, não se observou maior desenvolvimento de alterações neurocognitivas e/ou início de diabetes mellitus em pacientes submetidos a intervenção medicamentosa, hipóteses cogitadas no início da pesquisa.

Apesar dos benefícios citados com o uso do evolocumabe para a população de alto risco cardiovascular, o estudo FOURIER culminou com um número necessário para tratar (NNT) de 74 para evitar apenas 1 desfecho secundário. Isso traduz-se em um custo aproximado de 3 milhões de reais (preço da dose mensal no Brasil em abril de $2017 \simeq$ $\mathrm{R} \$ 1.500,00)$ para reduzir um evento dentre os seguintes: IAM, AVC ou morte por causa cardiovascular. No entanto, especialistas calculam que o estudo completo englobando um seguimento de 4 anos prevê um NNT= 25 para redução de desfechos secundários.

Outra limitação do estudo que deve ser destacada é o curto tempo de seguimento do ensaio clínico (2,2 anos) quando comparado a outros estudos com terapias antilipemiantes (média de 5 anos de seguimento), sendo impossivel prever eventuais efeitos adversos da medicação ao longo do tempo.

Dessa forma, conclui-se que a redução dos níveis séricos de LDL colesterol para um valor médio de $30 \mathrm{mg} / \mathrm{dl}$ observada no estudo FOURIER foi benéfica em pacientes de alto risco cardiovascular, sem trazer prejuízos adicionais a essa população. Porém, mais estudos com o evolocumabe ainda são necessários para corroborar seu verdadeiro custo-benefício a longo prazo. 


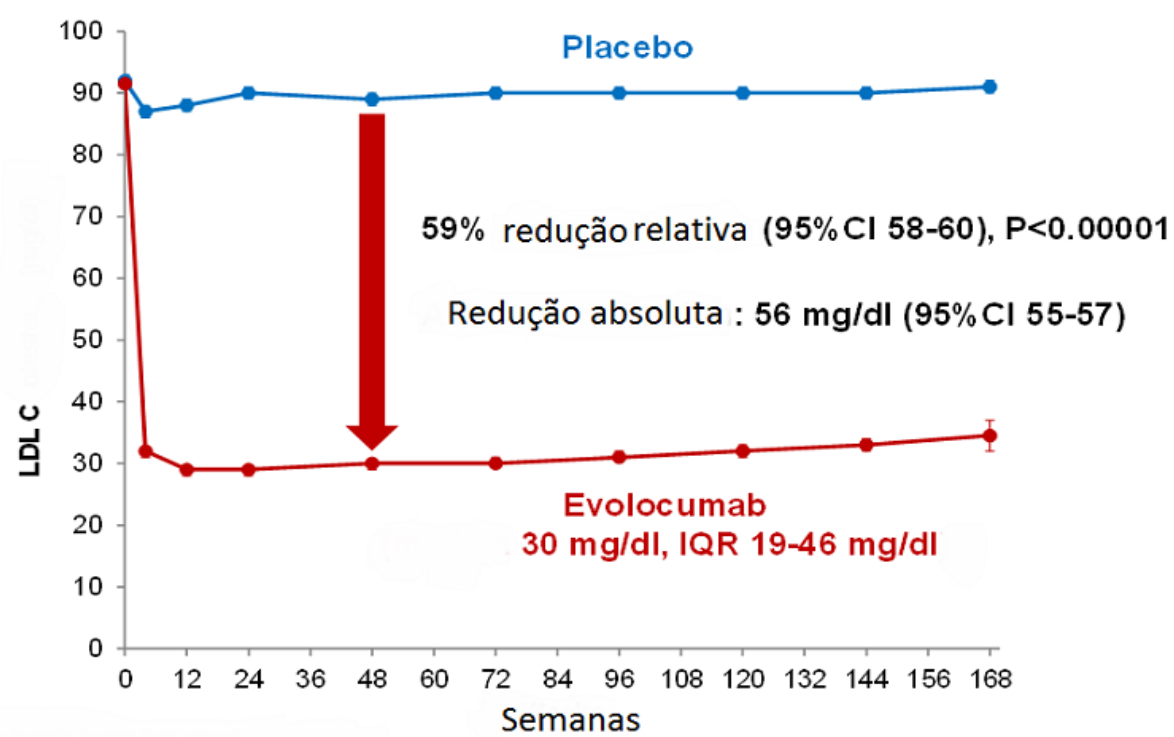

Figura 2: Níveis de LDL ao longo do estudo.

\section{REFERÊNCIAS}

1. Sabatine MS, Giugliano RP, Keech AC, et al. Evolocumab and clinical outcomes in patients with cardiovascular disease. N Engl J Med. 2017 Mar 17. doi: 10.1056/NEJMoa1615664. [Epub ahead of print]. 
